\title{
PRUNE BELLY SYNDROME ASSOCIATED WITH POSTERIOR URETHRAL VALVE AND BILATERAL POLYCYSTIC KIDNEY DISEASE: A CASE REPORT OF A NIGERIAN NEONATE
}

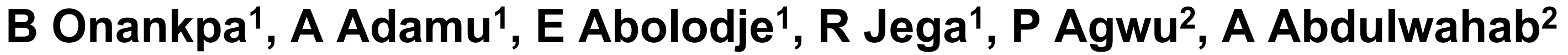

Title: Absence of anterior abdominal muscle with renal complication in a new born

Background: Prune-Belly syndrome is a rare congenital disorder that affects males and is associated with high neonatal mortality. This is the first case in literature from Northwestern part of the country.

Case Summary: A 7 - hour old term male neonate presented to the neonatal intensive care unit of our tertiary hospital with history of difficulty in breathing since birth. The mother, a 38-year old grand multiparous had history of maternal pyrexia at 4/12 gestation. She had no history of body rashes or exposure to radiation, and was not a known diabetic. The mother ingested traditional concoctions in early pregnancy in the form of powdered leaves for a febrile illness. An abdominal ultrasound did not reveal any fetal abnormality. No history of genetic anomaly in the family.

Examinations findings include respiratory distress, with wrinkling of the skin of the anterior abdominal wall, absence of anterior abdominal wall muscle and bilateral cryptorchidism. A diagnosis of Prune Belly syndrome with severe respiratory distress was made.

Abdominal ultrasound showed moderate hydronephrosis, with features of posterior urethral valve, bilateral polycystic kidney disease and non-demonstrable testicles. The patient had intra nasal oxygen, intravenous fluids and antibiotics. Patient developed features of acute urinary retention on account of which the urology team performed a vesicostomy. The baby was planned for dialysis due to worsening of renal function but the parents declined consent and requested for discharge on account of the fees. Patient died $18^{\text {th }}$ day on admission following sudden respiratory distress.

Discussion: There is the need for routine early antenatal ultrasonography to help in detecting the condition and associated renal anomaly early, for effective prenatal and postnatal management. 